\title{
Generalized Fokker-Planck equations and effective thermodynamics
}

\author{
Pierre-Henri Chavanis \\ Laboratoire de Physique Théorique, Université Paul Sabatier \\ 118 route de Narbonne, 31062 Toulouse Cedex 4, France \\ E-mail: chavanis@irsamc.ups-tlse.fr
}

\begin{abstract}
We introduce a new class of Fokker-Planck equations associated with an effective generalized thermodynamical framework. These equations describe a gas of Langevin particles in interaction. The free energy can take various forms which can account for anomalous diffusion, quantum statistics, lattice models... When the potential of interaction is long-ranged, these equations display a rich structure associated with canonical phase transitions and blow-up phenomena. In the limit of short-range interactions, they reduce to Cahn-Hilliard equations.
\end{abstract}

Key words: Generalized Fokker-Planck equations, generalized thermodynamics, stochastic processes, long-range interactions

\section{Introduction}

The statistical mechanics of systems with long-range interactions is currently a topic of active research. So far, most works have focused on the case of Hamiltonian systems of particles such as the $N$-stars problem, the $N$-vortex problem or the HMF model [1]. For these systems the energy is conserved and the proper statistical description is the microcanonical ensemble. The statistical evolution of the particles is described by kinetic equations such as the Landau equation in astrophysics [2] or the kinetic equation derived in [3] for point vortices. Since statistical ensembles are not equivalent for systems with long-range interactions, it may be of interest, at a conceptual level, to compare this microcanonical evolution with a canonical one. In that respect, we can consider a system of Brownian particles in interaction. These particles are in contact with a thermal bath that imposes its temperature. Therefore, they have a rigorous canonical structure and their statistical evolution is described by non-local Fokker-Planck equations $[4,5]$. 
In a different context, it has been shown that Tsallis generalized thermodynamics could be useful to interpret anomalous diffusion in complex systems and that the $q$-entropies are connected to nonlinear Fokker-Planck equations $[6,7]$. These equations can be derived from stochastic processes involving a multiplicative noise depending on the distribution function [8]. In fact, it is possible to construct a larger class of Fokker-Planck equations associated with a generalized thermodynamical framework encompassing Tsallis and Boltzmann entropies [9-12,5]. These generalized Fokker-Planck equations are obtained from standard ones by assuming that the diffusion coefficient is an arbitrary function $D(f)$ of the distribution function (the friction term can also depend on $f$ ). For these equations, there exists a Lyapunov functional, satisfying $\dot{F} \leq 0$, that can be interpreted as a generalized free energy. In this context, Tsallis free energy corresponds to a power-law dependence of the diffusion coefficient $D(f) \sim f^{q-1}$ and the $q$ parameter in Tsallis formalism is related to the exponent of anomalous diffusion.

In this paper, following [11], we introduce a new class of Fokker-Planck equations associated with a generalized free energy functional and an arbitrary potential of interaction. These equations connect the two topics discussed above: long-range interactions and generalized stochastic processes. Their mathematical and physical richness is considerable, as they exhibit a wide variety of canonical phase transitions and blow-up phenomena [13-17]. In the limit of short-range interactions, they reduce to Cahn-Hilliard equations.

\section{Generalized Fokker-Planck equations in phase space}

\subsection{Langevin particles in an external potential}

We consider a system of non-interacting Langevin particles evolving in a fixed external potential $\Phi_{\text {ext }}(\mathbf{r})$. We assume that the motion of each particle is described by the generalized stochastic process

$$
\frac{d \mathbf{r}}{d t}=\mathbf{v}, \quad \frac{d \mathbf{v}}{d t}=-\xi \mathbf{v}-\nabla \Phi_{\text {ext }}(\mathbf{r})+\sqrt{2 D f\left[\frac{C(f)}{f}\right]^{\prime}} \mathbf{R}(t),
$$

where $\mathbf{R}(t)$ is a white noise satisfying $\langle\mathbf{R}(t)\rangle=\mathbf{0}$ and $\left\langle R_{a}(t) R_{b}\left(t^{\prime}\right)\right\rangle=\delta_{a b} \delta(t-$ $t^{\prime}$ ) and $C$ is an arbitrary convex function, i.e. $C^{\prime \prime}>0$. Since the function in front of $\mathbf{R}(t)$ depends on $(\mathbf{r}, \mathbf{v})$, the last term in Eq. (1) can be interpreted as a multiplicative noise. We note, however, that it depends on $(\mathbf{r}, \mathbf{v})$ through the local density of particles $f(\mathbf{r}, \mathbf{v}, t)$. This corresponds to a back-reaction from the macroscopic dynamics. When $C(f)=\frac{1}{q-1}\left(f^{q}-f\right)$, Eq. (1) reduces 
to the stochastic process studied by Borland [8] in connexion with Tsallis thermodynamics.

Using standard methods, we can show that the stochastic process (1) leads to the generalized Fokker-Planck equation

$$
\frac{\partial f}{\partial t}+\mathbf{v} \cdot \frac{\partial f}{\partial \mathbf{r}}-\nabla \Phi_{e x t} \cdot \frac{\partial f}{\partial \mathbf{v}}=\frac{\partial}{\partial \mathbf{v}} \cdot\left\{D\left[f C^{\prime \prime}(f) \frac{\partial f}{\partial \mathbf{v}}+\beta f \mathbf{v}\right]\right\}
$$

where we have introduced a generalized inverse temperature $\beta=1 / T$ through the generalized Einstein relation $\xi=D \beta$. This equation can be put in the form

$$
\frac{\partial f}{\partial t}+L f=\frac{\partial}{\partial \mathbf{v}} \cdot\left[\xi f \frac{\partial}{\partial \mathbf{v}}\left(\frac{\delta F}{\delta f}\right)\right]
$$

where $L$ is the advection operator and $F$ is the generalized free energy

$$
F[f]=E-T S=\int f \frac{v^{2}}{2} d^{D} \mathbf{r} d^{D} \mathbf{v}+\int \rho \Phi_{e x t} d^{D} \mathbf{r}+T \int C(f) d^{D} \mathbf{r} d^{D} \mathbf{v}
$$

The first term in Eq. (4) is the kinetic energy, the second term is the potential energy and the third term is a generalized entropy $S=-\int C(f) d^{D} \mathbf{r} d^{D} \mathbf{v}$. It is easy to show that the dissipation of free energy can be expressed as

$$
\dot{F}=-\int \frac{D T}{f}\left[f C^{\prime \prime}(f) \frac{\partial f}{\partial \mathbf{v}}+\beta f \mathbf{v}\right]^{2} d^{D} \mathbf{r} d^{D} \mathbf{v}
$$

which is negative provided that $D>0$. Therefore, the generalized FokkerPlanck equation (2) satisfies a canonical H-theorem $\dot{F} \leq 0$. Hence, the free energy (4) is a Lyapunov functional. Finally, the stationary solutions of Eq. (2) are given by

$$
C^{\prime}(f)=-\beta\left(\frac{v^{2}}{2}+\Phi_{e x t}\right)-\alpha
$$

They extremize the free energy (4) at fixed mass and temperature. In addition, only minima of free energy are linearly stable via Eq. (2), see [11].

\subsection{Langevin particles in interaction}

We now consider a system of Langevin particles in interaction described by the $N$-body stochastic equations 


$$
\frac{d \mathbf{r}_{i}}{d t}=\mathbf{v}_{i}, \quad \frac{d \mathbf{v}_{i}}{d t}=-\xi \mathbf{v}_{i}-\nabla_{i} U\left(\mathbf{r}_{1}, \ldots, \mathbf{r}_{N}\right)+\sqrt{2 D f_{i}\left[\frac{C\left(f_{i}\right)}{f_{i}}\right]^{\prime}} \mathbf{R}_{i}(t),
$$

where $f_{i}=f\left(\mathbf{r}_{i}, \mathbf{v}_{i}, t\right)$ and $\mathbf{R}_{i}(t)$ is a white noise acting independently on the particles. The particles interact via the potential $U=\sum_{i<j} u\left(\mathbf{r}_{i}-\mathbf{r}_{j}\right)$ where $u\left(\mathbf{r}_{i}-\mathbf{r}_{j}\right)$ is an arbitrary binary potential depending only on the absolute distance between particles. When $C(f)=-f \ln f$, corresponding to the Boltzmann entropy, Eq. (7) describes a system of Brownian particles in interaction.

Starting from the $N$-body Fokker-Planck equation, using a Kramers-Moyal expansion and a mean-field approximation [4,5], we can derive from (7) the non-local generalized Kramers equation

$$
\frac{\partial f}{\partial t}+\mathbf{v} \cdot \frac{\partial f}{\partial \mathbf{r}}-\nabla \Phi \cdot \frac{\partial f}{\partial \mathbf{v}}=\frac{\partial}{\partial \mathbf{v}} \cdot\left\{D\left[f C^{\prime \prime}(f) \frac{\partial f}{\partial \mathbf{v}}+\beta f \mathbf{v}\right]\right\}
$$

where $\Phi$ is related to the density $\rho=\int f d^{D} \mathbf{v}$ by a relation of the form

$$
\Phi(\mathbf{r}, t)=\int \rho\left(\mathbf{r}^{\prime}, t\right) u\left(\mathbf{r}-\mathbf{r}^{\prime}\right) d^{D} \mathbf{r}^{\prime}
$$

Equation (8) can be written as Eq. (3) where, now, the free energy is given by

$$
F[f]=\int f \frac{v^{2}}{2} d^{D} \mathbf{r} d^{D} \mathbf{v}+\frac{1}{2} \int \rho \Phi d^{D} \mathbf{r}+T \int C(f) d^{D} \mathbf{r} d^{D} \mathbf{v}
$$

Furthermore, Eq. (5) remains valid so that $\dot{F} \leq 0$. Finally, the stationary states of Eq. (8) are determined by the integro-differential equation

$$
C^{\prime}(f)=-\beta\left\{\frac{v^{2}}{2}+\int f\left(\mathbf{r}^{\prime}, \mathbf{v}^{\prime}\right) u\left(\mathbf{r}-\mathbf{r}^{\prime}\right) d^{D} \mathbf{r}^{\prime} d^{D} \mathbf{v}^{\prime}\right\}-\alpha
$$

They extremize the free energy (10) at fixed mass and temperature. In addition, only minima are linearly stable via Eq. (8), see [11].

\subsection{The strong friction limit}

In the strong friction limit $\xi \rightarrow+\infty$, or equivalently for large times $t \gg \xi^{-1}$, the distribution function is given by

$$
C^{\prime}(f)=-\beta\left[\frac{v^{2}}{2}+\lambda(\mathbf{r}, t)\right]+O\left(\xi^{-1}\right)
$$


where $\lambda$ is related to the density $\rho$, using $\rho=\int f d^{D} \mathbf{v}=\rho(\lambda)$. Introducing the pressure $p=\frac{1}{D} \int f v^{2} d^{D} \mathbf{v}=p(\lambda)$ and eliminating $\lambda$, we find that the fluid is barotropic in the sense that $p=p(\rho)$, where the equation of state is entirely specified by the function $C(f)$. Furthermore, writing the hierarchy of moment equations [11] or using a formal Chapman-Enskog expansion [18], we can show that the evolution of the density is determined by the non-local generalized Smoluchowski equation

$$
\frac{\partial \rho}{\partial t}=\nabla \cdot\left[\frac{1}{\xi}(\nabla p+\rho \nabla \Phi)\right]
$$

which can be written explicitly as

$$
\frac{\partial \rho}{\partial t}=\nabla \cdot\left\{\frac{1}{\xi}\left[p^{\prime}(\rho) \nabla \rho+\rho \nabla \int u\left(\mathbf{r}-\mathbf{r}^{\prime}\right) \rho\left(\mathbf{r}^{\prime}, t\right) d^{D} \mathbf{r}^{\prime}\right]\right\}
$$

This equation can be put in the form

$$
\frac{\partial \rho}{\partial t}=\nabla \cdot\left[\frac{1}{\xi} \rho \nabla\left(\frac{\delta F}{\delta \rho}\right)\right]
$$

where $F$ is the free energy

$$
F[\rho]=\int \rho \int_{0}^{\rho} \frac{p\left(\rho^{\prime}\right)}{\rho^{\prime 2}} d \rho^{\prime} d^{D} \mathbf{r}+\frac{1}{2} \int \rho(\mathbf{r}, t) u\left(\mathbf{r}-\mathbf{r}^{\prime}\right) \rho\left(\mathbf{r}^{\prime}, t\right) d^{D} \mathbf{r} d^{D} \mathbf{r}^{\prime}
$$

It can be obtained from Eq. (10) by using the relation (12) valid in the strong friction limit $\xi \rightarrow+\infty$ to express $F[f]$ as a functional of $\rho[11,18]$. It is straightforward to show that

$$
\dot{F}=-\int \frac{1}{\xi \rho}(\nabla p+\rho \nabla \Phi)^{2} d^{D} \mathbf{r}
$$

which yields $\dot{F} \leq 0$. Therefore, the free energy (16) is a Lyapunov functional for the generalized Smoluchowski equation (14). Finally, the stationary solutions of Eq. (14) are determined by the condition of hydrostatic equilibrium

$$
\nabla p+\rho \nabla \Phi=\mathbf{0}
$$

which can be written explicitly as

$$
\frac{p^{\prime}(\rho)}{\rho} \nabla \rho=-\nabla \int \rho\left(\mathbf{r}^{\prime}\right) u\left(\mathbf{r}-\mathbf{r}^{\prime}\right) d^{D} \mathbf{r}^{\prime}
$$


They extremize the free energy (16) at fixed mass. In addition, only minima are linearly stable via Eq. (13).

\section{Generalized Fokker-Planck equations in physical space}

\subsection{Langevin particles in an external potential}

We consider a system of non-interacting Langevin particles described by the generalized stochastic process

$$
\frac{d \mathbf{r}}{d t}=-\xi \nabla \Phi_{e x t}(\mathbf{r})+\sqrt{2 D \rho\left[\frac{C(\rho)}{\rho}\right]^{\prime}} \mathbf{R}(t),
$$

where $\xi$ is the drift coefficient and $\mathbf{R}(t)$ is a white noise. The evolution of the density is described by the generalized Fokker-Planck equation

$$
\frac{\partial \rho}{\partial t}=\nabla \cdot\left\{D\left[\rho C^{\prime \prime}(\rho) \nabla \rho+\beta \rho \nabla \Phi_{e x t}\right]\right\}
$$

where we have introduced a generalized inverse temperature $\beta=1 / T$ through the generalized Einstein relation $\xi=D \beta$. This equation can be put in the form

$$
\frac{\partial \rho}{\partial t}=\nabla \cdot\left[\xi \rho \nabla\left(\frac{\delta F}{\delta \rho}\right)\right]
$$

where $F$ is the generalized free energy

$$
F[\rho]=E-T S=\int \rho \Phi_{e x t} d^{D} \mathbf{r}+T \int C(\rho) d^{D} \mathbf{r} .
$$

The rate of dissipation of free energy can be expressed as

$$
\dot{F}=-\int \frac{D T}{\rho}\left[\rho C^{\prime \prime}(\rho) \nabla \rho+\beta \rho \nabla \Phi_{e x t}\right]^{2} d^{D} \mathbf{r}
$$

which is negative provided that $D>0$. Therefore, the generalized FokkerPlanck equation (21) satisfies a sort of canonical H-theorem $\dot{F} \leq 0$. Hence, the free energy (23) is a Lyapunov functional. Finally, the stationary solutions of Eq. (21) are given by

$$
C^{\prime}(\rho)=-\beta \Phi_{\text {ext }}-\alpha .
$$


They extremize the free energy (23) at fixed mass and temperature. In addition, only minima of free energy are linearly stable via Eq. (21).

\subsection{Langevin particles in interaction}

We now consider a system of Langevin particles in interaction described by the $N$-body stochastic equations

$$
\frac{d \mathbf{r}_{i}}{d t}=-\xi \nabla_{i} U\left(\mathbf{r}_{1}, \ldots, \mathbf{r}_{N}\right)+\sqrt{2 D \rho_{i}\left[\frac{C\left(\rho_{i}\right)}{\rho_{i}}\right]^{\prime}} \mathbf{R}_{i}(t),
$$

where $\rho_{i}=\rho\left(\mathbf{r}_{i}, t\right)$ and $\mathbf{R}_{i}(t)$ is a white noise. The potential of interaction $U$ is defined as above. Starting from the $N$-body Fokker-Planck equation, using a Kramers-Moyal expansion and a mean-field approximation [5], we can derive from Eq. (26) the non-local generalized Smoluchowski equation

$$
\frac{\partial \rho}{\partial t}=\nabla \cdot\left\{D\left[\rho C^{\prime \prime}(\rho) \nabla \rho+\beta \rho \nabla \Phi\right]\right\}
$$

where $\Phi$ is related to the density $\rho$ by a relation of the form (9). Equation (27) can be written as (22) where, now, the free energy is given by

$$
F=\frac{1}{2} \int \rho \Phi d^{D} \mathbf{r}+T \int C(\rho) d^{D} \mathbf{r} .
$$

Furthermore, Eq. (24) remains valid so that $\dot{F} \leq 0$. Finally, the stationary states of Eq. (27) are determined by the integro-differential equation

$$
C^{\prime}(\rho)=-\beta \int \rho\left(\mathbf{r}^{\prime}, t\right) u\left(\mathbf{r}-\mathbf{r}^{\prime}\right) d^{D} \mathbf{r}^{\prime}-\alpha
$$

They extremize the free energy (28) at fixed mass and temperature. In addition, only minima are linearly stable via Eq. (27).

\subsection{Connexion with Cahn-Hilliard equations}

If we now consider the case of short-range interactions, it is possible to expand the potential

$$
\Phi(\mathbf{r}, t)=\int u\left(\mathbf{r}^{\prime}\right) \rho\left(\mathbf{r}+\mathbf{r}^{\prime}\right) d^{D} \mathbf{r}^{\prime}
$$


in Taylor series for $\mathbf{r}^{\prime} \rightarrow \mathbf{0}$. Introducing the notations

$$
a=\int u(|\mathbf{x}|) d^{D} \mathbf{x} \quad \text { and } \quad b=\frac{1}{D} \int u(|\mathbf{x}|) x^{2} d^{D} \mathbf{x}
$$

we obtain to second order

$$
\Phi(\mathbf{r}, t)=a \rho(\mathbf{r}, t)+\frac{b}{2} \Delta \rho(\mathbf{r}, t) .
$$

In that limit, the free energy (28) takes the form

$$
F[\rho]=-\frac{b}{2} \int\left\{\frac{(\nabla \rho)^{2}}{2}+V(\rho)\right\} d^{D} \mathbf{r}
$$

where we have set $V(\rho)=-(2 T / b) C(\rho)-(a / b) \rho^{2}$. This is the usual expression of the Landau free energy. In general $b$ is negative so we have to minimize this functional integral. For systems with short-range interactions, the conservative equation (27) becomes

$$
\frac{\partial \rho}{\partial t}=\nabla \cdot\left\{\frac{b \xi}{2} \rho \nabla\left(\Delta \rho-V^{\prime}(\rho)\right)\right\}
$$

This is the Cahn-Hilliard equation which has been extensively studied in the theory of phase ordering kinetics. Its stationary solutions describe "domain walls". We can view therefore Eq. (27) as a generalization of the Cahn-Hilliard equation to the case of systems with long-range interactions.

\section{Conclusion}

In this paper, we have introduced a wide class of generalized Fokker-Planck equations and we have shown that they were associated with a consistent thermodynamical formalism. We have derived these equations from a particular stochastic process involving a special form of multiplicative noise, but they can also arise from other types of microscopic models. In our point of view, generalized Fokker-Planck equations are "effective equations" trying to take into account "hidden constraints" in complex media that are difficult to formalize [12]. Since these equations decrease a functional similar to a generalized free energy, this leads naturally to a notion of "effective thermodynamics". This effective approach is not in contradiction with usual thermodynamics. This is just a heuristic approach trying to deal with complex situations in which 
standard thermodynamics is difficult to implement. We can either use ordinary thermodynamics (Boltzmann) and try to take into account microscopic constraints or keep only the most accessible constraints (mass, energy,...) and introduce an "effective entropy". This heuristic approach may be useful in certain occasions when an exact description of the system's dynamics is difficult to implement. Generalized thermodynamics is justified when all the accessible microstates are not equiprobable due to additional microscopic constraints. These constraints can be due to quantum mechanics (Pauli exclusion principle), to the existence of a lattice, to hard sphere effects, to a fractal structure of phase space, etc. We emphasize that our formalism is valid for more general functionals than the Tsallis entropy. Tsallis entropy forms, however, an important class of functionals associated with polytropic distributions and power-laws. These distributions generate a natural form of self-confinement that can be of interest in nonextensive systems. However, it was our interest here to show that a more general formalism could be developed consistently. A notion of "generalized thermodynamics", with a different presentation and a different motivation, has been developed independently by Kaniadakis [9], Frank [10] and Naudts [19].

The non-local Fokker-Planck equations introduced in this paper can be of interest in different fields of physics, with various interpretations. In the usual case where they are associated with the Boltzmann entropy, they describe a gas of Brownian particles in interaction $[4,5]$. Therefore, they constitute the canonical counterpart of the microcanonical kinetic equations (Boltzmann, Landau, Lenard-Balescu,...) describing Hamiltonian systems of particles in interaction $[2,3]$. They are thus interesting at a conceptual level to test dynamically situations of ensemble inequivalence for systems with long-range interactions. For example, the "isothermal collapse" of self-gravitating Brownian particles described by the Smoluchowski-Poisson system [13-16] can be compared with the "gravothermal catastrophe" of the Hamiltonian $N$-stars system described by the Landau-Poisson system (or by the orbit averaged Fokker-Planck equation). Similarly, the Smoluchowski-Poisson system for Brownian point vortices can be contrasted with the kinetic equation obtained in [3] from the Hamiltonian dynamics. They exhibit a very different behaviour illustrating the inequivalence of statistical ensembles.

At a practical level, the generalized Fokker-Planck equations presented in this paper and in [11] can be used as numerical algorithms to compute explicitly nonlinearly dynamically stable stationary solutions of the Vlasov and Euler equations. In that context, the generalized entropy $S=-\int C(f) d^{D} \mathbf{r} d^{D} \mathbf{v}$ is interpreted as a Casimir functional or a $H$-function [20]. Its maximization at fixed energy and mass (or circulation) determines a condition of dynamical stability, not a condition of thermodynamical stability. The fact that this variational principle looks similar to the variational principle that occurs in thermodynamics is essentially a thermodynamical analogy [11]. Since the gen- 
eralized Fokker-Planck equations introduced in [11] increase the functional $S$ while conserving $E$ and $M$ (or $\Gamma$ ), they can be used as physical algorithms to construct arbitrary nonlinearly dynamically stable stationary solutions of the Vlasov (or Euler) equation. Such stationary solutions can arise in practice as a result of a (possibly incomplete) violent relaxation, leading to a metaequilibrium state on the coarse-grained scale $[21,11,12]$.

Finally, non-local drift-diffusion equations isomorphic to the SmoluchowskiPoisson system have a real interest in biology in relation with the concept of chemotaxis (see [17] for a description of the analogy between self-gravitating Brownian particles and bacterial populations). Now, it has been noted that the diffusion of bacteries is anomalous in general so that the diffusion coefficient which appears in the drift-diffusion equation depends on the density. This leads to a notion of "effective" generalized thermodynamics. This is simply because the true dynamics is so complex that it is replaced by a simple equation with an "ad hoc" diffusion coefficient whose specific form has to be deduced from experiments (this illustrates what we mean by "hidden constraints"). Accordingly, different forms of "generalized free energy" functionals can arise in biology depending on the context.

In conclusion, the generalized Fokker-Planck equations presented in this paper and in [11] can be of considerable interest, in particular in the case of long-range interactions where they exhibit a rich variety of canonical phase transitions. A systematic study of this new class of equations has been initiated in $[13-17]$.

\section{References}

[1] Dynamics and thermodynamics of systems with long range interactions, edited by Dauxois, T., Ruffo, S., Arimondo, E. and Wilkens, M. Lecture Notes in Physics, Springer (2002).

[2] H.E. Kandrup, ApJ 244, 316 (1981).

[3] P.H. Chavanis, Phys. Rev. E 64, 026309 (2001).

[4] N. Martzel and C. Aslangul, J. Phys. A 34, 11225 (2001).

[5] P.H. Chavanis, cond-mat/0409641.

[6] A.R. Plastino and A. Plastino, Physica A 222, 347 (1995)

[7] C. Tsallis and D.J. Bukman, Phys. Rev. E 54, R2197 (1996).

[8] L. Borland, Phys. Rev. E 57, 6634 (1998)

[9] G. Kaniadakis, Physica A 296, 405 (2001).

[10] T.D. Frank, Physics Lett. A 290, 93 (2001).

[11] P.H. Chavanis, Phys. Rev. E 68, 036108 (2003).

[12] P.H. Chavanis, Physica A 332, 89 (2004).

[13] P.H. Chavanis, C. Rosier and C. Sire, Phys. Rev. E 66, 036105 (2002).

[14] C. Sire and P.H. Chavanis, Phys. Rev. E 66, 046133 (2002). 
[15] C. Sire and P.H. Chavanis, Phys. Rev. E 69, 066109

[16] P.H. Chavanis and C. Sire, Phys. Rev. E 69, 016116 (2004).

[17] P.H. Chavanis, M. Ribot, C. Rosier and C. Sire, Banach Center Publ. 66, 103 (2004).

[18] P.H. Chavanis, P. Laurençot and M. Lemou, Physica A, 341, 145 (2004).

[19] Naudts, Physica A 332, 279 (2004).

[20] S. Tremaine, M. Hénon and D. Lynden-Bell, Mon. Not. R. Astron. Soc. 219, 285 (1986).

[21] P.H. Chavanis, J. Sommeria and R. Robert, Astrophys. J. 471, 385 (1996). 